\title{
The Clinical Evaluation and Use of Sleep Studies in Neurological Practice
}

\author{
Kathryn Williams $^{1}$ • Anita Valanju Shelgikar ${ }^{1}$
}

Published online: 17 April 2015

(C) Springer International Publishing AG 2015

\begin{abstract}
The polysomnogram is a very important diagnostic tool in the evaluation of many sleep disorders. We will review common neurologic presenting complaints, their relationship to sleep disorders, neurologic conditions that are at high risk for co-morbid sleep disorders, and the utility of using the polysomnogram for their evaluation. Additionally, we will outline the indications for in-laboratory polysomnography versus out-of-center sleep apnea testing.
\end{abstract}

Keywords Polysomnogram $\cdot$ Headache $\cdot$ Memory impairment $\cdot$ Seizures $\cdot$ Stroke $\cdot$ Multiple sclerosis $\cdot$ Alpha synucleinopathies $\cdot$ Obstructive sleep apnea $\cdot$ REM sleep behavior disorder

\section{Introduction}

Sleep and circadian disorders are reported in $25-30 \%$ of the general population [1]. Obstructive sleep apnea (OSA) is a chronic condition characterized by episodes of partial (hypopnea) or complete (apnea) upper airway occlusion during sleep. In the general population, 1 in 5 adults will have OSA but will have minimal to no symptoms. It is less common for patients with OSA to have daytime impairment

This article is part of the Topical Collection on Sleep and Neurological Disorders

Kathryn Williams

boyleke06@gmail.com

1 Sleep Disorders Center, Department of Neurology, University of Michigan, 1500 East Medical Center Drive, Ann Arbor, MI 481095845, USA symptoms, coined OSA syndrome, in that only occurs in 1 in 20 adults [2]. Patients with specific neurologic conditions such as stroke [3], cluster headaches [4], multiple sclerosis [5•], and neuromuscular disease [6] are at higher risk of having co-morbid OSA than the general population.

Other sleep disorders, including central hypersomnias and sleep-related movement disorders, insomnias, circadian rhythm sleep-wake disorders, parasomnias, and sleep-related epilepsy may also be seen in patients with neurological diseases [7]. Negative impact of sleep disorders on quality of life and daytime functioning has been reported in neurological disease [8-10]. Diagnosis and treatment of co-morbid sleep disorders may facilitate improved quality of life for these patients.

We will discuss common presenting symptoms and neurologic conditions that warrant consideration for a possible sleep disorder that can and should be evaluated by a sleep study.

\section{Clinical Evaluation}

A sleep medicine history and physical exam are essential to determine the presence of an underlying sleep disorder. Getting a collateral history from a bed partner or family may fill in gaps in the patient's recollection of their sleep habits [11]. The American Academy of Sleep Medicine (AASM) [12] has published recommendations on specific symptoms that should be inquired about during a sleep medicine history. These include snoring, apneas, nocturnal choking or gasping, restlessness, and excessive daytime sleepiness. These symptoms generally relate to sleep-disordered breathing, but it is equally as important to screen for symptoms of other co-morbid sleep disorders. These symptoms would include dream enactment, which may suggest rapid eye movement (REM) sleep behavior disorder (RBD) or a non-REM parasomnia; cataplexy, or 
hypnagogic or hypnopompic hallucinations, which may indicate narcolepsy; excessive worry about sleep with mind racing, hyperarousal, and difficulty napping during the day suggestive of insomnia; or leg discomfort with the urge to move, as seen in restless leg syndrome.

The physical exam is equally important in helping to decide the likelihood of underlying sleep-disordered breathing. A comprehensive physical exam with special attention to neck size ( $>16 \mathrm{~cm}$ in women, $>17 \mathrm{~cm}$ in men) [13], nasal airflow (obtained by the patient occluding one nostril at a time), nasal septal deviation, jaw positioning (retrognathia), tonsil hypertrophy, narrow or high-arched hard palate, and Mallampati score (III or IV) (Fig. 1) [11, 14].

\section{Clinical Scenarios}

\section{Headache}

A 55-year-old female presents to neurology clinic with headaches. She describes them as dull in character, frontal, holocephalic, and wakes up with them most mornings. She has had migraines previously but these headaches are different. She has recently gone through menopause and has gained $30 \mathrm{lb}$ in the last 3 years.

Headache is a common symptom of sleep-disordered breathing. Classically, morning headaches are thought to be associated with sleep-disordered breathing. However, headache in isolation is not a specific symptom for obstructive sleep apnea [15]. Morning headaches are common in other conditions that may also impact sleep such as anxiety, depression, circadian rhythm disorder, and chronic insomnia [16]. While it can be challenging to identify that a headache is more likely to be associated with a sleep disorder [17••], recognition of certain characteristics may help with this determination. For instance, morning headaches may represent sleep apnea headaches. The definition of a sleep apnea headache is one that is present on awakening, occurs in patients with diagnosed obstructive sleep apnea (apnea hypopnea index (AHI) $\geq 5$ events/ h), worsened at the onset of sleep apnea or improved with treatment of sleep apnea or bilateral, and has at least one of the three criteria: recurs on $>15$ days a month, resolves within $4 \mathrm{~h}$, or has specific characteristics of a headache pressing in quality not accompanied by photophobia, phonophobia, or nausea [18]. This type of headache has been reported to be present in $11.8 \%$ of patients with obstructive sleep apnea and in $4.8 \%$ of the general population without diagnosed obstructive sleep apnea [19].

Two additional headache types have a historical association with obstructive sleep apnea. A hypnic headache generally occurs from 1 to $3 \mathrm{a} . \mathrm{m}$. and is most common in elderly females [20]. Polysomnographic data collected by Holle et al. [21] suggests that these types of headaches do not have an association with sleep-disordered breathing. Secondly, cluster headaches have been found to have an eight times higher risk of having co-morbid obstructive sleep apnea. Nobre et al. [4] reported a brisk drop in the incidence of OSA in patients with headaches when the BMI was $<25 \mathrm{~kg} / \mathrm{m}^{2}$ or the age was $<40$ years. With these observations, the authors recommended only evaluating for obstructive sleep apnea in headache patients with a BMI $>25 \mathrm{~kg} / \mathrm{m}^{2}$ or age $>40$ years.

\section{Worsening Control of Seizures}

A 56-year-old male with a history of epilepsy is brought into the emergency room in status epilepticus. He has had good control of his seizures for the last 20 years but was having two seizures a week in the last 2 months. He has been taking his anti-epileptic medication regularly. He does report frequent nocturnal awakenings in the last 6 months and he is unaware of the cause.

Patients with partial epilepsy are twice as likely to have a sleep disorder as control patients [22]. Jain and Glauser [23•] reviewed anti-epileptic drugs and found that some increased decreased sleep latency (carbamazepine, clobazam, pregabalin, gabapentin, and tiagabine) while others aggravated daytime sleepiness (phenobarbital, valproic acid, and highdose levetiracetam). Foldvary-Schaefer et al. [24] determined that increasing age and anti-epileptic drug load raise the risk of obstructive sleep apnea in a patient with epilepsy due to possible weight gain, inactivity, and decreased upper airway tone.

Standard polysomnography recording includes six electroencephalography (EEG) leads. In epilepsy patients with suspected sleep-disordered breathing, use of expanded EEG monitoring with polysomnography should be determined on a case-by-case basis. Bubrick et al. [25] suggest in their review that adding full EEG can help identify potential causes of excessive daytime sleepiness in specific epilepsy patients. Extended EEG montage with polysomnography can also be useful in the general population with undiagnosed nocturnal behaviors or with daytime changes in awareness without clear causes. These observations should be interpreted with caution as they were based off of a small population and were derived from a cohort. According to the AASM, the only specific indication for extended EEG monitoring is to clarify paroxysmal arousals or other sleep disruptions that are thought to be seizure related when standard EEG monitoring is unrevealing [12].

Sudden unexplained death in epilepsy (SUDEP) is "the sudden, unexpected, witnessed or unwitnessed, non-traumatic, and non-drowning death of patients with epilepsy with or without evidence of a seizure, excluding documented status epilepticus, and in which postmortem examination does not reveal a structural or toxicological cause of death" [26]. The majority of SUDEPs are not witnessed; however, the etiologies of the rare witnessed events are categorized into cardiac- 
Fig. 1 Modified Mallampati classification of oropharyngeal visualization (Huang et al. [14])
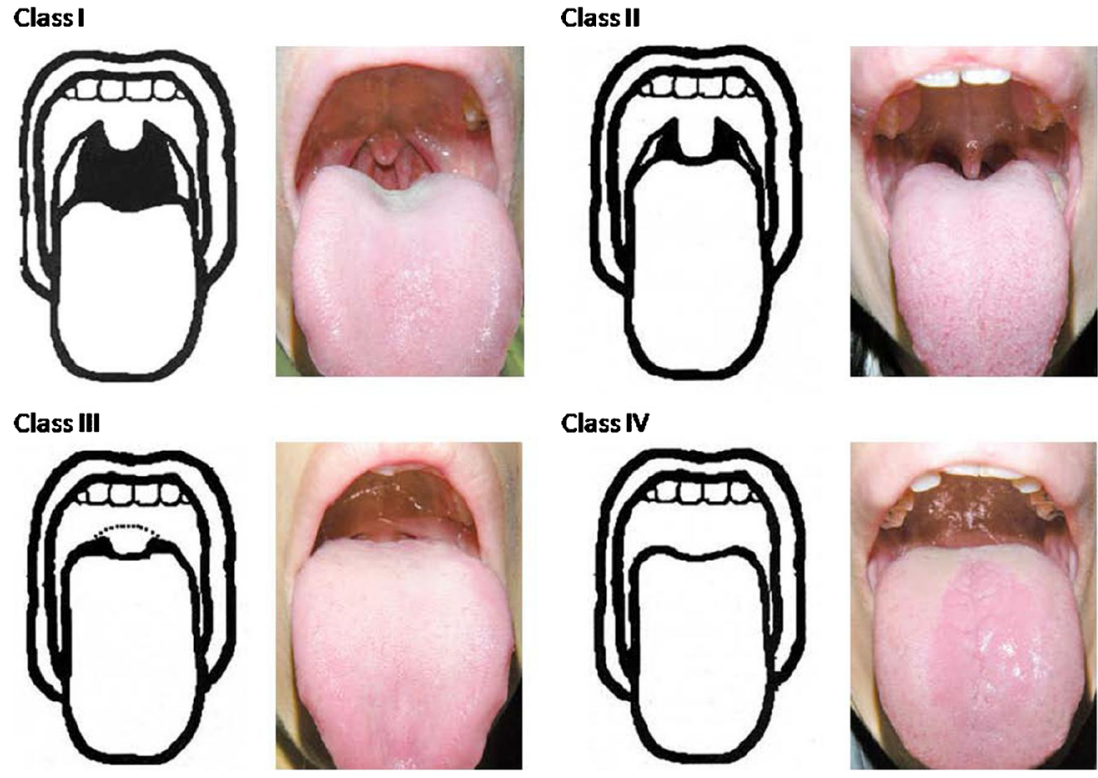

autonomic, respiratory, and cerebral shut down. Nobili et al. [27] reviewed the literature and found that SUDEPs were related to sleep in 60 to $95 \%$ of the case report series. Periictal respiratory changes have been described as a central or obstructive event occurring before, during or after a seizure [28]. Respiratory events can lead to autonomic instability [29] which is implicated in SUDEP [27, 30].

\section{Memory Impairment}

A 75-year-old male presents with worsening memory and difficulty with concentration. He has not been sleeping well for over 10 years and has multiple awakenings at night, some due to nocturia but otherwise unexplained.

The relationship between mild cognitive impairment, dementia, and sleep-disordered breathing is complicated and not fully identified. In the Apnea Positive Pressure Long-term Efficacy Study (APPLES), middle age patients, with an average age of 52 years, showed a mild increase in cognitive function with continuous positive airway pressure (CPAP) in patients with obstructive sleep apnea [31••]. Recent studies have identified nocturnal hypoxia and decreased slow-wave sleep as risk factors for gray matter changes, but not for Alzheimer's disease or Lewy body dementia [32]. To date, evidence regarding the impact of obstructive sleep apnea treatment on cognitive function in the elderly is inconclusive. In our experience, elderly patients with dementia often have difficulty with adherence to nightly use of positive airway pressure therapy, which limits the benefits of obstructive sleep apnea treatment. This clinical experience is supported by a meta-analysis completed by Kylstra et al. [33], who concluded that CPAP use led to only a small improvement in cognitive function. Interestingly, the average number of hours of CPAP used overall was $4.5 \mathrm{~h}$ per night. This meets most insurance compliance standards; however, half of the studies reviewed had an average of less than $4 \mathrm{~h}$ per night.

\section{Multiple Sclerosis}

A 39-year-old female is seen in follow-up for her diagnosis of primary progressive multiple sclerosis (MS). Her main complaint is fatigue.

Fatigue is a debilitating symptom for the general population but has been identified as one of the top causes for poor quality of life in patients with MS [34]. Fatigue in MS is often multi-factorial secondary to depression, disease-modifying agents, high lesion load, and sleep disorders [35]. In the general population, the presenting complaint of fatigue may be as important as that of sleepiness when evaluating for OSA [36]. Kaminska et al. [37] reported a significant relationship between severe fatigue and OSA in patients with MS. Interestingly, they also reported a strong relationship between severe fatigue on the Fatigue Severity Scale (FSS) and severe OSA. Treatment of sleep-disordered breathing also results in an improvement on the Modified Fatigue Impact Scale (MFIS) by approximately $35 \%$ [38]. Patients compliant with CPAP reported less fatigue, tiredness, lack of energy, and sleepiness compared to those patients with OSA who were not compliant with CPAP therapy [39].

Patients with multiple sclerosis are also at higher risk of central sleep apnea, respiratory muscle weakness, and sleeprelated hypoventilation secondary to demyelinating lesions in the brainstem [40]. It is important to include carbon dioxide monitoring $\left(\mathrm{CO}_{2}\right)$, in the form of end-tidal $\mathrm{CO}_{2}$ monitoring, to exclude hypoventilation as a cause for daytime sleepiness.

Similarities in human leukocyte antigens exist between MS and narcolepsy. These similarities support the theory of autoimmunity involved in the pathogenesis of both diseases [41]. 
In the evaluation of excessive daytime sleepiness or hypersomnolence in a patient with multiple sclerosis, consideration can be given to the possibility of narcolepsy even in the setting of co-morbid sleep-disordered breathing. The multiple sleep latency test (MSLT), preceded by a baseline polysomnogram, is used to make the diagnosis of narcolepsy. During the MSLT, patients are given five opportunities to fall asleep in a bed in a dark room. The patient is given $20 \mathrm{~min}$ to fall asleep and, then once asleep, is allowed to sleep for $15 \mathrm{~min}$ [42]. The average time it takes to fall asleep during the nap trials, known as the mean sleep latency, is compared to normative data to determine pathologic sleepiness.

\section{Neuromuscular Disease}

A 62-year-old male recently diagnosed with amyotrophic lateral sclerosis (ALS) presented to neurology clinic for worsening daytime fatigue and difficulty breathing at night. He has started sleeping in the recliner in the living room because he finds it very difficult to breathe when he is completely supine. He denies shortness of breath during the day.

In patients with known or suspected neuromuscular disease, it is recommended that an in-lab polysomnogram be used for diagnosis of sleep-disordered breathing. The addition of end-tidal carbon dioxide $\left(\mathrm{ETCO}_{2}\right)$ monitoring is also important in the diagnosis and treatment of sleep-related hypoventilation caused by neuromuscular weakness of the respiratory muscles. The diagnosis is made when $\mathrm{ETCO}_{2}$, or transcutaneous $\mathrm{CO}_{2}\left(\mathrm{TCO}_{2}\right)$ levels are greater than $55 \mathrm{mmHg}$ for $10 \mathrm{~min}$ or $\mathrm{a} \geq 10 \mathrm{mmHg}$ increase in $\mathrm{ETCO}_{2} / \mathrm{TCO}_{2}$ during sleep (in comparison to an awake supine value) to a value exceeding $50 \mathrm{mmHg}$ for $\geq 10 \mathrm{~min}$ [43]. Once the diagnosis of sleep-related hypoventilation has been made, the AASM has published specific titration criteria for initiation of positive airway pressure [44]. A review of the treatment with nocturnal mechanical ventilation in patients with neuromuscular disorders concluded that there was prolonged survival, decreased unplanned hospitalizations, and improvement in quality of life [45]. There are no current guidelines on the frequency of which patients on noninvasive positive pressure ventilation should be evaluated for efficacy of therapy. However, home monitoring with $\mathrm{ETCO}_{2}$ alone has been shown to be effective in assessing nocturnal hypoventilation and compliance with therapy [46].

\section{Stroke}

A 65-year-old male is hospitalized for an ischemic stroke. His nurse is concerned about his observed pauses in his breathing. The patient's wife denies that this was occurring at home. He had a sleep study 1 year prior, due to history of snoring, which did not show obstructive sleep apnea.

\section{Indications}

- High pre-test probability for moderate to severe obstructive sleep apnea

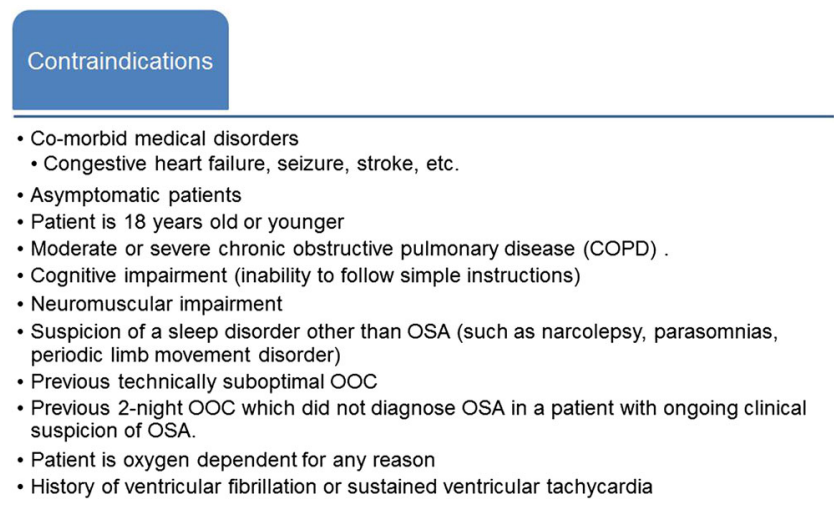

Fig. 2 Indications and contraindications for home sleep apnea testing

OSA has been shown to be an independent risk factor for ischemic stroke [3]. A recent systematic review and metaanalysis by Xie et al. [47••] reported a concern for obstructive sleep apnea's role in serious adverse outcomes in patients with recent stroke. The importance of evaluating stroke patients for untreated OSA is well documented in the literature. The

Table 1 Indications for sleep testing

An indication for polysomnogram?

\begin{tabular}{ll}
\hline Symptoms & \\
Snoring & Yes \\
Apneas & \\
Nocturnal choking/gasping & \\
Restlessness & \\
Excessive daytime sleepiness & \\
Medical conditions & \\
Systemic hypertension & Yes, with symptoms listed above \\
Obesity & \\
Congestive heart failure & \\
Significant cardiac & \\
arrhythmias & \\
Neurological conditions ${ }^{\mathrm{a}}$ & \\
Migraines & Not at a higher risk for OSA \\
Cluster headaches & Yes, high association with OSA \\
Hypnic headaches & Not at a higher risk OSA \\
Epilepsy & Higher risk for OSA with high-dose \\
& AED \\
Multiple sclerosis & Yes \\
Stroke & Yes \\
$\alpha$-Synucleinopathies & With a history for nocturnal behaviors \\
Stroke & Yes, with daytime symptoms \\
&
\end{tabular}

${ }^{\mathrm{a}}$ These medical and neurologic conditions have a strong association with sleep disorders. However, patients should also have accompanying symptoms, but a lower threshold for testing with polysomnogram may be considered given the higher likelihood of co-morbid sleep disorder 
composition of sleep-disordered breathing, including central and obstructive apnea, may change in the first few months following a stroke [48]. While the correlation of OSA and stroke has been well represented in the literature, the evidence for treatment of OSA in the acute post-stroke period is underwhelming [49].

\section{$\alpha$-Synucleinopathies}

A 72-year-old male presents with hand tremor, decreased smell, constipation, and soft speech. His wife has slept in the guest bedroom for the past 5 years because he has while asleep he shouts as if he is in an argument. On occasion, he has made punching movements, which has raised concerns about injury to his wife beside him. He is a mild-tempered man, and his wife reports that his sleep-related behavior is out of character for him and very different from his demeanor while awake.

Rapid eye movement (REM) sleep behavior disorder (RBD) is a parasomnia characterized by loss of muscle atonia with dream enactment often composed of violent behaviors, such as hitting or kicking, or verbal outbursts [18]. The International Classification of Sleep Disorders [18] criteria require that the REM sleep without atonia be observed on a polysomnogram to fulfill the diagnosis of RBD. The complex motor behaviors or vocalizations may be observed on the night of the study or may be clinically suspected to occur out of REM sleep. Historical characteristics would include the events occurring later in the night due to increased percentage of REM sleep occurring in the second half of the night.

Dream-enactment behavior is not likely to be the presenting symptom to a general neurology clinic. However, it is a very important symptom in the evaluation of possible synucleinopathies as it is very likely an early symptom in a neurodegenerative disorder [50]. Postuma et al. [51 • $]$ reviewed the risk of development of a neurodegenerative disorder in the setting of idiopathic RBD and reported that the risk in current literature ranges from 38 to $82 \%$; the risk increases as time from onset of RBD increases. Those patients that had nocturnal behaviors prior to the start of antidepressant medication may carry similar risks as opposed to those patients who had no prior history of behaviors suggestive of RBD [52].

\section{Which Sleep Study to Order?}

Once the clinical suspicion is finalized, the next step is to select the appropriate testing. We have outlined the AASM practice parameters [53 ${ }^{\circ}$ for appropriate use of out-of-center (OOC, also referred to home/portable sleep testing) testing for sleep apnea (Fig. 2) and in-lab polysomnogram (Table 1). Additionally, as previously discussed, the role of MSLT becomes important in diagnosing narcolepsy. Additional monitoring techniques such as end-tidal carbon dioxide $\left(\mathrm{ETCO}_{2}\right)$ and expanded EEG montage are helpful for diagnosing hypoventilation seen in progressive neuromuscular conditions and for concern for nocturnal seizures, respectively.

\section{Conclusion}

Sleep disorders are vast in their presentation but carry significant health implications. The polysomnogram is a valuable tool that is used to elucidate a multitude of sleep disorders, though clinicians need to understand indications for an attended polysomnography and home sleep apnea testing. Diagnosis and treatment of sleep disorders may facilitate improved control or early identification of neurological diseases, along with improved quality of life.

\section{Compliance with Ethics Guidelines}

Conflict of Interest Kathryn Williams and Anita Valanju Shelgikar declare that they have no conflict of interest.

Human and Animal Rights and Informed Consent This article does not contain any studies with human or animal subjects performed by any of the authors.

\section{References}

Papers of particular interest, published recently, have been highlighted as:

- Of importance

-• Of major importance

1. National institutes of health: National center on sleep disorders research. Sleep disorders research plan. www.nhlbi.nih.gov/ health/prof/sleep/201101011NationalSleepDisordersResearchPlanDHHSPublication11-7820.pdf. Updated Published Nov 2011.

2. Young T, Peppard PE, Gottlieb DJ. Epidemiology of obstructive sleep apnea: a population health perspective. Am J Respir Crit Care Med. 2002;165(9):1217-39.

3. Yaggi HK, Concato J, Kernan WN, Lichtman JH, Brass LM, Mohsenin V. Obstructive sleep apnea as a risk factor for stroke and death. N Engl J Med. 2005;353(19):2034-41.

4. Nobre ME, Leal AJ, Filho PM. Investigation into sleep disturbance of patients suffering from cluster headache. Cephalalgia. 2005;25(7):488-92.

5. Braley TJ, Segal BM, Chervin RD. Sleep-disordered breathing in multiple sclerosis. Neurology. 2012;79(9):929-36. This article is an excellent overview of sleep-disordered breathing in patients with multiple sclerosis.

6. Chokroverty S. Sleep and breathing in neuromuscular disorders. Handb Clin Neurol. 2011;99:1087-108.

7. Dyken ME, Afifi AK, Lin-Dyken DC. Sleep-related problems in neurologic diseases. Chest. 2012;141(2):528-44. 
8. Barone P, Antonini A, Colosimo C, et al. The PRIAMO study: a multicenter assessment of nonmotor symptoms and their impact on quality of life in Parkinson's disease. Mov Disord. 2009;24(11): 1641-9.

9. Brass SD, Li CS, Auerbach S. The underdiagnosis of sleep disorders in patients with multiple sclerosis. J Clin Sleep Med. 2014;10(9):1025-31.

10. Alanis-Guevara I, Pena E, Corona T, Lopez-Ayala T, Lopez-Meza E, Lopez-Gomez M. Sleep disturbances, socioeconomic status, and seizure control as main predictors of quality of life in epilepsy. Epilepsy Behav. 2005;7(3):481-5.

11. Shelgikar AV, Chervin R. Approach to and evaluation of sleep disorders. Continuum (Minneap Minn). 2013;19(1 Sleep Disorders): 32-49.

12. Kushida CA, Littner MR, Morgenthaler T, et al. Practice parameters for the indications for polysomnography and related procedures: an update for 2005. Sleep. 2005;28(4):499-521.

13. Davies RJ, Stradling JR. The relationship between neck circumference, radiographic pharyngeal anatomy, and the obstructive sleep apnoea syndrome. Eur Respir J. 1990;3(5):509-14.

14. Huang HH, Lee MS, Shih YL, Chu HC, Huang TY, Hsieh TY. Modified Mallampati classification as a clinical predictor of peroral esophagogastroduodenoscopy tolerance. BMC Gastroenterol. 2011;11:12.

15. Aldrich MS, Chauncey JB. Are morning headaches part of obstructive sleep apnea syndrome? Arch Intern Med. 1990;150(6):1265-7.

16. Ohayon MM. Prevalence and risk factors of morning headaches in the general population. Arch Intern Med. 2004;164(1):97-102.

17.• Singh NN, Sahota P. Sleep-related headache and its management. Curr Treat Options Neurol. 2013;15(6):704-22. This is a comprehensive review of the relationship of headaches and sleep.

18. American Academy of Sleep Medicine. International classification of sleep disorders, 3rd ed. Darien, IL: American Academy of Sleep Medicine, 2014.

19. Kristiansen HA, Kvaerner KJ, Akre H, Overland B, Sandvik L, Russell MB. Sleep apnoea headache in the general population. Cephalalgia. 2012;32(6):451-8.

20. Evers S, Goadsby PJ. Hypnic headache: clinical features, pathophysiology, and treatment. Neurology. 2003;60(6):905-9.

21. Holle D, Wessendorf TE, Zaremba S, et al. Serial polysomnography in hypnic headache. Cephalalgia. 2011;31(3):286-90.

22. de Weerd A, de Haas S, Otte A, et al. Subjective sleep disturbance in patients with partial epilepsy: a questionnaire-based study on prevalence and impact on quality of life. Epilepsia. 2004;45(11): $1397-404$.

23. Jain SV, Glauser TA. Effects of epilepsy treatments on sleep architecture and daytime sleepiness: an evidence-based review of objective sleep metrics. Epilepsia. 2014;55(1):26-37. This is a thorough review of anti-epileptic medications and their impact on sleep.

24. Foldvary-Schaefer N, Andrews ND, Pornsriniyom D, Moul DE, Sun Z, Bena J. Sleep apnea and epilepsy: who's at risk? Epilepsy Behav. 2012;25(3):363-7.

25. Bubrick EJ, Yazdani S, Pavlova MK. Beyond standard polysomnography: advantages and indications for use of extended 10-20 EEG montage during laboratory sleep study evaluations. Seizure. 2014;23(9):699-702.

26. Nashef L. Sudden unexpected death in epilepsy: terminology and definitions. Epilepsia. 1997;38(11 Suppl):S6-8.

27. Nobili L, Proserpio P, Rubboli G, Montano N, Didato G, Tassinari CA. Sudden unexpected death in epilepsy (SUDEP) and sleep. Sleep Med Rev. 2011;15(4):237-46.

28. Blum AS. Respiratory physiology of seizures. J Clin Neurophysiol. 2009;26(5):309-15.

29. Vanninen E, Tuunainen A, Kansanen M, Uusitupa M, Lansimies E. Cardiac sympathovagal balance during sleep apnea episodes. Clin Physiol. 1996;16(3):209-16.
30. Nayak C, Sinha S, Nagappa M, Thennarasu K, Taly AB. Lack of heart rate variability during apnea in patients with juvenile myoclonic epilepsy (JME). Sleep Breath. 2015.

31.• Kushida CA, Nichols DA, Holmes TH, et al. Effects of continuous positive airway pressure on neurocognitive function in obstructive sleep apnea patients: the apnea positive pressure long-term efficacy study (APPLES). Sleep. 2012;35(12):1593-602. This study is important in evaluating the utility of CPAP on neurocognitive function in patients with sleep apnea.

32. Gelber RP, Redline S, Ross GW, et al. Associations of brain lesions at autopsy with polysomnography features before death. Neurology. 2015;84(3):296-303.

33. Kylstra WA, Aaronson JA, Hofman WF, Schmand BA. Neuropsychological functioning after CPAP treatment in obstructive sleep apnea: a meta-analysis. Sleep Med Rev. 2013;17(5):3417.

34. Janardhan V, Bakshi R. Quality of life in patients with multiple sclerosis: the impact of fatigue and depression. J Neurol Sci. 2002;205(1):51-8.

35. Veauthier C, Paul F. Sleep disorders in multiple sclerosis and their relationship to fatigue. Sleep Med. 2014;15(1):5-14.

36. Chervin RD. Sleepiness, fatigue, tiredness, and lack of energy in obstructive sleep apnea. Chest. 2000;118(2):372-9.

37. Kaminska M, Kimoff RJ, Benedetti A, et al. Obstructive sleep apnea is associated with fatigue in multiple sclerosis. Mult Scler. 2012;18(8):1159-69.

38. Veauthier C, Gaede G, Radbruch H, Gottschalk S, Wernecke KD, Paul F. Treatment of sleep disorders may improve fatigue in multiple sclerosis. Clin Neurol Neurosurg. 2013;115(9):1826-30.

39. Chotinaiwattarakul W, O'Brien LM, Fan L, Chervin RD. Fatigue, tiredness, and lack of energy improve with treatment for OSA. J Clin Sleep Med. 2009;5(3):222-7.

40. Fleming WE, Pollak CP. Sleep disorders in multiple sclerosis. Semin Neurol. 2005;25(1):64-8.

41. Younger DS, Pedley TA, Thorpy MJ. Multiple sclerosis and narcolepsy: possible similar genetic susceptibility. Neurology. 1991;41(3):447-8.

42. Littner MR, Kushida C, Wise M, et al. Practice parameters for clinical use of the multiple sleep latency test and the maintenance of wakefulness test. Sleep. 2005;28(1):113-21.

43. Berry RB, Brooks R, Gamaldo CE, et al. The AASM manual for the scoring of sleep and associated events: rules, terminology, and technical specifications. Version 21st ed. Darien: American Academy of Sleep Medicine; 2014.

44. Berry RB, Chediak A, Brown LK, et al. Best clinical practices for the sleep center adjustment of noninvasive positive pressure ventilation (NPPV) in stable chronic alveolar hypoventilation syndromes. J Clin Sleep Med. 2010;6(5):491-509.

45. Annane D, Orlikowski D, Chevret S. Nocturnal mechanical ventilation for chronic hypoventilation in patients with neuromuscular and chest wall disorders. Cochrane Database Syst Rev. 2014;12: CD001941.

46. Kim SM, Park KS, Nam H, et al. Capnography for assessing nocturnal hypoventilation and predicting compliance with subsequent noninvasive ventilation in patients with ALS. PLoS One. 2011;6(3):e17893.

47.• Xie W, Zheng F, Song X. Obstructive sleep apnea and serious adverse outcomes in patients with cardiovascular or cerebrovascular disease: a PRISMA-compliant systematic review and meta-analysis. Med (Baltimore). 2014;93(29):e336. This article highlights the concerns for serious short- and long-term complications post-stroke in patients with untreated severe obstructive sleep apnea.

48. Parra O, Arboix A, Bechich S, et al. Time course of sleep-related breathing disorders in first-ever stroke or transient ischemic attack. Am J Respir Crit Care Med. 2000;161(2 Pt 1):375-80. 
49. Tomfohr LM, Hemmen T, Natarajan L, et al. Continuous positive airway pressure for treatment of obstructive sleep apnea in stroke survivors: what do we really know? Stroke. 2012;43(11):311823.

50. Gagnon JF, Postuma RB, Mazza S, Doyon J, Montplaisir J. Rapideye-movement sleep behaviour disorder and neurodegenerative diseases. Lancet Neurol. 2006;5(5):424-32.

51.• Postuma RB, Gagnon JF, Montplaisir JY. REM sleep behavior disorder: from dreams to neurodegeneration. Neurobiol Dis. 2012;46(3):553-8. This article summarizes the importance of identifying RBD as an early marker of possible $\alpha$ synucleinopathies.

52. Postuma RB, Gagnon JF, Tuineaig M, et al. Antidepressants and REM sleep behavior disorder: isolated side effect or neurodegenerative signal? Sleep. 2013;36(11):1579-85.

53. Collop NA, Tracy SL, Kapur V, et al. Obstructive sleep apnea devices for out-of-center (OOC) testing: technology evaluation. J Clin Sleep Med. 2011;7(5):531-48. These guidelines for home sleep apnea testing are important to be aware of when considering the type of testing that is indicated for patients. 